\section{The first implementation of the NIMH FAST-FAIL approach to psychiatric drug development}

\author{
Andrew D. Krystal, Diego A. Pizzagalli, Sanjay J. Mathew, Gerard Sanacora, \\ Richard Keefe, Allen Song, Joseph Calabrese, Andrew Goddard, \\ Wayne Goodman, Sarah H. Lisanby, Moria Smoski, Richard Weiner, \\ Dan Iosifescu, John Nurnberger Jr, Steven Szabo, James Murrough, \\ Anantha Shekhar and William Potter
}

The low probability of success has resulted in such high costs for developing muchneeded novel drugs for central nervous system (CNS) disorders that several major pharmaceutical companies have stopped investment in this area ${ }^{1}$. A key contributor is early-phase trial methods, which are slow and frequently mislead companies into pursuing extremely costly unsuccessful phase III studies $^{1}$. The NIMH FAST-FAIL initiative sought to address this problem by supporting early-phase drug development methodologies designed to lower the risk of failure in large clinical trials. Under the auspices of the NIMH Fast Mood and Anxiety Disorders Program (Fast-MAS), we were the first to successfully implement this approach, applying it to assess the potential of $\kappa$-opioid receptor (KOR) antagonism for treating anhedonia cross-diagnostically. In this article, we share the methodology we developed and the lessons we learned in the hopes that this will facilitate future applications of the 'fast-fail' approach, thus accelerating much-needed drug development.

\section{Rationale for the fast-fail approach}

The fast-fail approach is based on the premise that most candidate drugs will ultimately fail to be approved for their intended clinical application, so the goal should be to eliminate them earlier and at lower cost than is currently possible. It requires early-phase methodology to be modified so that it provides a more reliable basis for 'go/no-go' decision-making rooted in objective measures (TABLE 1).

As a road map for implementing this approach, the NIMH developed the New Experimental Medicine Studies: Fast-Fail Trials Program, which funded our Fast-MAS Program (see Related links). The key starting point for this effort was identifying the drug target to study. To this end, we first had to establish a set of requirements that candidate targets must meet in order to be effectively developed with a fast-fail approach. After obtaining extensive input from industry, government and academic scientists, we generated the following requirements:

- a compelling body of preclinical (and, if available clinical) research establishing that engaging the target was likely to have a brain effect that might prove therapeutic;

- a robust method for measuring engagement of the target by a compound;

- a compound that specifically engages the target with sufficient preclinical safety data to support human trials;

- a biomarker of a brain effect with therapeutic potential that could serve as the outcome measure for a proof of mechanism (POM) study.

\section{Selection of KOR antagonism}

We assiduously followed these requirements despite facing practical implementation challenges in order to garner the payoff of a rigorous implementation of the fast-fail approach: improved capacity to establish the promise of a target with confidence in early-phase human studies of limited size.

One challenge we faced was that, for many targets, there are no robust means of measuring target engagement. A leading CNS target engagement measure, quantification of receptor occupancy with positron emission tomography (PET), has the limitation that radioligands are not available for many targets. Moreover, for agonists and partial agonists, clinical effects may occur at relatively low levels of receptor occupancy, leaving uncertainty as to what level of occupancy is required for robust target engagement ${ }^{2}$. As a result, we limited our target search to those targets for which a well-established PET ligand was available and to receptor antagonists for which we could be confident that an appropriate goal for ensuring clinical effects was near-complete receptor occupancy. On this basis alone, KOR antagonism was a leading candidate as there

Table 1 | Summary of the 'fast-fail' approach to early-phase psychiatric drug development

\section{Key step in fast-fail approach}

Develop/select a biomarker that reflects activity of the experimental compound at the neurobiological target

Use this target engagement biomarker to determine doses of a selective drug that robustly engages the target for use in subsequent studies

Conduct phase lla studies testing the specific proof of mechanism hypothesis that engaging the target achieves an effect on the brain thought to mediate the anticipated clinical effect

Proceed to studies with clinical end points only if proof of mechanism is established; otherwise, 'fail' the drug

PET, positron emission tomography.

\section{Comments}

Ideally, this would be PET receptor occupancy or other imaging-based probes of target engagement (for example, functional magnetic resonance imaging, magnetic resonance spectroscopy)

Where the measure is PET receptor occupancy, robust engagement would be indicated by near-complete receptor occupancy (occupancy levels that are in the asymptotic portion of the dose-occupancy curve)

The rationale is that effects on the brain are closer to the direct neurobiological effects of the drug than to the clinical effects, and, as a result are likely to be detectable more reliably and with a smaller number of subjects than the clinical effects. This addresses the problem that phase II studies with clinical end points have produced misleading results because they are nearly always underpowered

Demonstrating that engaging the target activates the mechanisms thought to mediate clinical effects de-risks proceeding to larger clinical studies. It provides reassurance that effects on clinical end points found in phase Ilb studies are likely to be mediated by those hypothesized neural mechanisms rather than the result of bias and other non-specific effects that do not reflect an actual therapeutic effect of engaging the target, which have been the bane of psychiatric drug development 
existed a validated, specific KOR PET tracer, $\left[{ }^{11}\right.$ C]PKAB (LY2879788), which had been used to establish near saturation of receptor occupancy for a $10 \mathrm{mg}$ dosage of JNJ67953964 (previously known as CERC-501 and LY2456302) ${ }^{3}$. Another favourable aspect of KOR antagonism was that JNJ-67953964 is a high-affinity, selective KOR antagonist with favourable pharmacological and safety profiles ${ }^{4}$.

Another challenge was the limited availability of robust biomarkers for psychiatric disorders that could be used as POM study outcome measures ${ }^{5}$. In order to increase biomarker availability, we worked within the NIMH Research Domain Criteria Project (RDoC) dimensional diagnostic framework, which, unlike the traditional Diagnostic and Statistical Manual (DSM) framework, is neuroscience based and associated with phenotypic entities more likely to have associated biomarkers ${ }^{6}$. For KOR antagonism, there was a compelling body of preclinical work indicating a likely effect on a clinical entity, anhedonia (as instantiated as the RDoC constructs 'reward responsiveness', 'reward learning' and 'reward valuation'), a core symptom of major depressive disorder that cuts across traditional DSM diagnoses ${ }^{7}$. This choice was further strengthened by the availability of a biomarker for assessing outcome in a POM study. Striatal activation to reward-predicting cues, as assessed with functional magnetic resonance imaging (fMRI) in conjunction with the monetary incentive delay task (MID), was previously found to reflect neural activity that mediates clinical effects related to anhedonia and is correlated with striatal dopamine release as assessed by $\mathrm{PET}^{7-9}$.

Focusing on RDoC reward-related subdomains also enabled us to incorporate a novel feature into the design of our POM study which will be beneficial for future studies: we defined primary and secondary outcome variables across units of analysis (brain circuitry, behaviour and self-report) that were a priori hypothesized to be linked to the mechanism of action of the drug. This, in turn, enabled us to evaluate effect sizes for the different units of analyses, expecting that those that are closer to the effects of the drug (that is, brain circuitry) will show larger effect sizes than those that are more removed (that is, self-report measures). Such information can, in turn, be used to power future studies on the same or similar mechanisms.

\section{Trial conduct}

We carried out a FAST-MAS POM trial testing the hypothesis that KOR antagonism would enhance ventral striatal activation during anticipation of reward in the MID (ClinicalTrials.gov identifier: NCT02218736). This was a phase IIa, 8-week, double-blind, parallel-group, placebo-controlled study of JNJ-67953964 (at a fixed oral dose of $10 \mathrm{mg}$ per day) versus placebo, conducted at six US academic medical centres in patients meeting DSM-V mood or anxiety disorder diagnostic criteria who also had anhedonia (Snaith Hamilton Pleasure Scale Score $\geq 20$ ).

An important aspect of the trial conduct was investment in the standardization of measures across sites to improve signal-tonoise ratio and build a foundation for replication. We took a number of steps beyond providing sites with written materials and multiple presentations of detailed methods to standardize assessments and outcome measures across sites. For the primary outcome measure (MID fMRI) and the computerbased behavioural measures, we also provided one-on-one expert consultation to site personnel. Furthermore, for the MID fMRI, we provided all sites (which differed in the maker and models of MRI devices used) with the same EPrime files for running the MID fMRI protocol and required all sites to obtain and upload to our central fMRI core an agar phantom scan prior to being approved to proceed with the study and regularly throughout the study. Following the FIRST-BIRN multisite fMRI study quality assurance protocol, our fMRI core analysed the agar phantom scans and all study subject scans by reviewing the raw data, generating estimates of signalto-noise ratio and signal-to-fluctuation-noise ratio and assessing the adequacy of these parameters ${ }^{10}$. The finding of artefacts or problems and failure to meet adequacy criteria led to contact with the sites and implementation of corrective action. Lastly, on-site help was offered and was required by one of the sites. With these steps, we were able to achieve acceptable inter-site reliability on our key measures.

The trial was intended to be the basis for 'go/no go' decision-making. Establishment of POM would de-risk subsequent phase III trials and support proceeding with the development of a KOR antagonist for treating anhedonia. Conversely, in the absence of POM, it would be inadvisable to proceed because of the increased risk of a negative phase III trial even if therapeutic effects were found on clinical outcomes, as such effects could be due to factors other than engagement of neural reward circuitry, including nonspecific effects and bias. Importantly, a negative POM study could also reflect limitations of the primary outcome measure. Another key requirement of the fast-fail approach is that the POM study primary outcome be established to be sufficiently sensitive to detect the effect of interest so that a negative outcome provides a definitive indication of a failure to affect the brain circuitry of interest.

This trial was recently completed and successfully established POM for KOR antagonism as a treatment for anhedonia. This makes it more likely that significant effects found in adequately powered trials of KOR antagonists with clinical anhedonia endpoints would not be due to non-specific effects or bias, and supports proceeding with such trials.

\section{Conclusions}

It is hoped that our effort will serve as a model for how to implement the fast-fail approach. Features that are likely to be most helpful include:

- specification of requirements that targets must meet in order to be effectively developed with the fast-fail approach;

- strategies for identifying targets meeting those requirements;

- strategies for carrying out fast-fail studies that incorporate a small number of outcome variables across units of analysis - brain circuitry (primary), behaviour (secondary) and self-report (secondary) that were a priori hypothesized to be linked to the drug mechanism of action;

- implementing strategies for optimizing standardization across sites.

Our experience also highlighted a key limitation of the fast-fail approach. It is only possible to study a circumscribed set of targets owing to the limited means available for reliably establishing target engagement and for determining the impact of target engagement on neural function. Expanding the tools available for these purposes will facilitate future fast-fail trials and thereby improve the reliability and speed of early-phase CNS drug development.

Andrew D. Krystal ${ }^{1,2}$, Diego A. Pizzagalli ${ }^{3}$ Sanjay J. Mathew ${ }^{4}$, Gerard Sanacora ${ }^{5}$, Richard Keefe ${ }^{2}$, Allen Song ${ }^{2}$, Joseph Calabrese ${ }^{6}$, Andrew Goddard ${ }^{7}$, Wayne Goodman 4 , Sarah H. Lisanby ${ }^{2,7}$, Moria Smoski ${ }^{2}$, Richard Weiner ${ }^{2}$, Dan losifescu ${ }^{8}$, John Nurnberger Jr ${ }^{9}$ Steven Szabo ${ }^{2}$, James Murrough ${ }^{10}$, Anantha Shekhar ${ }^{9}$ and William Potter ${ }^{7}$

'University of California San Francisco, San Francisco, CA, USA.

${ }^{2}$ Duke University School of Medicine, Durham, NC, USA.

${ }^{3}$ McLean Hospital, Harvard Medical School, Belmont, MA, USA.

${ }^{4}$ Baylor School of Medicine, Houston, TX, USA.

${ }^{5}$ Yale University School of Medicine New Haven, CT, USA

${ }^{6}$ Case Western Reserve School of Medicine, Cleveland, $\mathrm{OH}, \mathrm{USA}$.

${ }^{7}$ National Institute of Mental Health, Bethesda, $M D, U S A$. 


\section{CORRESPONDENCE}

${ }^{8}$ New York University School of Medicine, New York,

NY, USA.

${ }^{9}$ Indiana University School of Medicine, Indianapolis,

IN, USA.

${ }^{10}$ Mount Sinai School of Medicine, New York, NY, USA.

*e-mail: andrew.krystal@ucsf.edu

doi: $10.1038 /$ nrd.2018.222

Published online 28 Dec 2018

1. Kesselheim, A. S., Hwang, T. J. \& Franklin, J. M Two decades of new drug development for central nervous system disorders. Nat. Rev. Drug Discov. 14, 815-816 (2015).

2. Passchier, J. et al. Measuring drug-related receptor occupancy with positron emission tomography. Methods 27, 278-286 (2002)

3. Zheng, M. Q. et al. Synthesis and evaluation of ${ }^{11} \mathrm{CLY} 2795050$ as a $\mathrm{k}$-opioid receptor antagonist radiotracer for PET imaging. J. NuCl. Med. 54, 455-463 (2013)
4. Lowe, S. L. et al. Safety, tolerability, and pharmacokinetic evaluation of single- and multipleascending doses of a novel kappa opioid receptor antagonist LY2456302 and drug interaction with ethanol in healthy subjects. J. Clin. Pharmacol. 54 968-978 (2014)

5. Wiedemann, K. Biomarkers in development of psychotropic drugs. Dialogues Clin. Neurosci. 13, 225-234 (2011).

6. Insel, T. et al. Research domain criteria (RDoC): toward a new classification framework for research on mental disorders. Am. J. Psychiatry. 67, 748-751 (2010).

7. Carlezon, W. A. Jr \& Krystal, A. D. Kappa-opioid antagonists for psychiatric disorders: From bench to clinical trials. Depress. Anxiety 33, 895-906 (2016).

8. Stoy, M. et al. Hyporeactivity of ventral striatum towards incentive stimuli in unmedicated depressed patients normalizes after treatment with escitalopram. J. Psychopharmacol. 26, 677-688 (2012).

9. Schott, B. H. et al. Mesolimbic functional magnetic resonance imaging activations during reward anticipation correlate with reward-related ventral striatal dopamine release. J. Neurosci. 28, 14311-14319 (2008).
10. Friedman, L. \& Glover, G. H. Report on a multicenter fMRI quality assurance protocol. J. Magn. Reson. Imaging. 23, 827-839 (2006).

\section{Acknowledgements}

Research reported in this publication was supported by the National Institute of Mental Health of the National Institutes of Health under Contract HHS N271 2012000006 I. The content is solely the responsibility of the authors and does not necessarily represent the official views of the National Institutes of Health. D.A.P. was partially supported by R37MH068376 and R01MH101521.

\section{Competing interests}

The authors declare competing interests: see Web version for details.

RELATED LINKS

FAST Fast-Fail Trials: https://www.nimh.nih.gov/researchpriorities/research-initiatives/fast-fast-fail-trials.shtml

ALL LINKS ARE ACTIVE IN THE ONLINE PDF 